\title{
Live Attenuated Influenza Virus (LAIV) Induces Different Mucosal T Cell Function In Nonsmokers And Smokers
}

\author{
Katie Horvath ${ }^{1}$, Missy Brighton, Margaret Herbst ${ }^{2,3}$, Terry L. Noah ${ }^{2,3}$, and llona Jaspers ${ }^{1,2,3}$ \\ ${ }^{1}$ Curriculum in Toxicology, The University of North Carolina at Chapel Hill, Chapel Hill, NC \\ 27599-7127 \\ ${ }^{2}$ Center for Environmental Medicine, Asthma and Lung Biology, The University of North Carolina \\ at Chapel Hill, Chapel Hill, NC 27599-7127 \\ ${ }^{3}$ Department of Pediatrics, The University of North Carolina at Chapel Hill, Chapel Hill, NC \\ 27599-7127
}

\begin{abstract}
Smokers are more susceptible to respiratory infections, including influenza. To explore the effect of smoking on influenza-induced responses within the nasal mucosa, we have developed a protocol using inoculation with live attenuated influenza virus (LAIV) vaccine followed by sampling of the nasal mucosa. Mucosal cell populations were harvested through superficial biopsy of the nasal inferior turbinate pre and post LAIV inoculation and analyzed using flow cytometry. The majority of nasal biopsy CD45+ immune cells at baseline were CD3+ T lymphocytes. Following LAIV, these lymphocytes increased in nonsmokers but not in smokers. A subset of individuals was negative for helper T cell marker CD4 and cytotoxic T cell marker CD8 but positive for the $\gamma \delta \mathrm{T}$ cell receptor (TCR). Increases in $\gamma \delta \mathrm{TCR}+$ cells were greater in nonsmokers, than in smokers. Thus, LAIV-induced changes in CD3 T as well as $\gamma \delta \mathrm{T}$ lymphocyte percentages are suppressed in smokers compared to nonsmokers.
\end{abstract}

\section{Keywords}

Cigarette smoke; influenza; gamma delta T cells; nasal mucosa

\section{Introduction}

Environmental stimuli, such as inhaled pollutants and pathogens, can initiate immune responses in the nasal mucosa that impact overall respiratory health. Cigarette smoke in particular is a risk factor for respiratory infections including influenza virus. Despite a clear epidemiological link, the mechanisms underlying this susceptibility are unknown. Although deficiencies in innate immune nasal defenses have been suggested [1-3], the effects of smoking on adaptive lymphocyte responses are not well understood. During influenza infection, the activation of the adaptive immune system, including $\mathrm{T}$ and $\mathrm{B}$ lymphocytes, is necessary to clear the initial infection through antibody and cell mediated cytolysis of virus infected cells, and to generate a memory response. The adaptive $\mathrm{T}$ cell response culminates

\footnotetext{
(C) 2011 Elsevier Inc. All rights reserved.
}

Publisher's Disclaimer: This is a PDF file of an unedited manuscript that has been accepted for publication. As a service to our customers we are providing this early version of the manuscript. The manuscript will undergo copyediting, typesetting, and review of the resulting proof before it is published in its final citable form. Please note that during the production process errors may be discovered which could affect the content, and all legal disclaimers that apply to the journal pertain. 
in the formation of specific memory $\mathrm{T}$ and $\mathrm{B}$ cells that patrol the circulation to mount an immediate adaptive immune response upon subsequent encounters with the same antigen. While there do not appear to be differences in influenza-specific antibody production in smokers [4-6], the effect of smoking status on the ability of T cells to mount proper mucosal and memory immune responses is unknown. We have shown previously that cigarette smoke exposure to nasal epithelial cells decreases their ability to activate dendritic cells (DC) during influenza infection. Because T cell activity is influenced by DC activation, we hypothesized that smokers have decreased $\mathrm{T}$ cell activation following influenza infection compared to nonsmokers.

We have previously used live attenuated influenza virus (LAIV), an influenza vaccine that induces immune responses to generate viral memory without causing actual disease [7-9], to demonstrate that smokers have markers of increased viral replication in nasal washes following inoculation [3]. LAIV generates secretion of lymphokines, including IP-10, within the nasal passages that are suppressed in smokers [3]. These lymphokines are important in attracting lymphocytes, such as natural killer (NK) cells and T lymphocytes to the nasal airways following viral infection. While animal models have shown that virus specific CD8 T cells migrate to the lower airways by day 7 post LAIV [10] the effects of LAIV on human nasal tissue itself with regards to the T cell trafficking are unknown. To assess the effects of cigarette smoking on LAIV-induced lymphocyte activation within the nasal mucosa, we administered LAIV to healthy young individuals as described previously [3] and quantified immune cells in superficial nasal biopsies using flow cytometry.

\section{Materials and Methods}

\section{Study Design}

This was a prospective longitudinal study characterizing responses to LAIV in between cohorts of healthy young adult smokers and nonsmokers. Subjects were inoculated with the 2009-2010 LAIV strain. The study design was as described before $[3,16]$. Baseline measurements were done at a screening visit, approximately 2-3 weeks prior to inoculation with LAIV. On Day 0 subjects received a standard nasal inoculum of LAIV (FluMist ${ }^{\circledR}$, MedImmune, Gaithersburg, MD; administered by study nurse according to manufacturer's instructions) in both nostrils, then returned on day 1 post-LAIV for nasal biopsy in the left nostril and day 4 post-LAIV for nasal biopsy in the right nostril. Serial nasal lavages were obtained on Days 0-4 and Day 9 post LAIV to assay for T cell populations.

\section{Study Subject Description}

Subjects were identified as described before [3] and included healthy young adults between $18-35$. Nonsmokers $(n=11)$ were 7 males and 4 females with an average age of $21.5 \pm 0.8$ and an average BMI of 25.5 \pm 1.9 . Smokers $(\mathrm{n}=12)$ were 10 males and 2 females with an average age of $25.3 \pm 1.3$ and an average BMI of $24.8 \pm 1.7$. . Subject exposure history questionnaires were used to estimate cigarette smoke exposure. Smokers smoked an average of $11.2 \pm 1.6$ cigarettes per day with nonsmokers reporting no cigarette use. Urine cotinine was measured by ELISA (Bio-Quant, Inc., San Diego, CA) and expressed as a ratio to creatinine, measured by a colorimetric assay (Oxford Biomedical Research, Rochester Hills, MI). Ratio of urine cotinine to creatine was 55.2 \pm 8.0 in smokers and $0.0 \pm 0.0$ in nonsmokers. Exclusion criteria were as described before [3]. No serious adverse events occurred among subjects completing the protocol. Informed consent was obtained from all subjects and the protocol was approved by the UNC Biomedical Institutional Review Board. 


\section{Nasal Biopsy}

Nasal biopsies were harvested from the inferior turbinates of both nostrils using a RhinoProbe cuvette and were placed in RPMI media. Biopsies were pelleted and treated with $15 \mu \mathrm{g} / \mathrm{ml}$ DNAse-1 (Roche, Indianapolis, IN) and $5 \mu \mathrm{g} / \mathrm{ml}$ pronase (Sigma, St. Louis, MO) for 30 minutes. FBS (Invitrogen) at a final concentration of 10\% was added to the cell pellet and filtered with a $40 \mathrm{uM}$ cell strainer (BD Falcon, San Jose, CA) to remove larger epithelial cells. The cell pellet was resuspended in $100 \mu \mathrm{l}$ of flow cytometry buffer (PBS plus $1 \%$ heat inactivated FBS plus $0.09 \%$ sodium azide) and stained with surface markers CD45 APC-cy7, CD3 PerCP, CD4 APC, CD8 PE, CD69 APC (BDBiosciences), and pan $\gamma \delta$ TCR FITC (Biolegend, San Diego, CA) for 20 minutes at room temperature. Cells were washed with flow buffer, fixed with $0.5 \%$ paraformaldehyde, and analyzed on a BDLSRII flow cytometer within 24 hours. T cells were identified by gating using forward scatter (FSC) and side scatter (SSC) settings and gating on cells staining positive for CD45 and CD3.

\section{Statistical Analysis}

The effects of LAIV on nasal biopsy responses were analyzed using a Wilcoxon signed rank test. Intergroup differences were determined using a Mann Whitney Test. Data were shown as mean $\pm \mathrm{SEM}$.

\section{Results}

\section{T cells Are Present In Nasal Biopsies At Baseline}

Nasal biopsies were harvested at screen day and processed for flow cytometry. The majority of nasal biopsy cells were epithelial cells, characterized by high forward scatter fluorescence and high side scatter fluorescence (Figure 1A). A population of epithelial cells in gate P2 had high nonspecific staining for immune markers that did not exceed isotype matched controls (data not shown). A distinct population of immune cells was identified based on high CD45 staining and low side scatter fluorescence in gate P1 as shown in Figure 1B. CD45+ cells were positive for T cell marker CD3 (Figure 1C) but were negative for activated lymphocyte marker CD69 (Figure 1D), helper T cell marker CD4 (Figure 1E), and cytotoxic T cell marker CD8 (Figure 1F). CD3 T cells were not identified in nasal lavage populations following LAIV (data not shown for this sample set, see previous analysis of nasal lavage cell populations following LAIV in nonsmokers and smokers) [16].

\section{LAIV Inoculation Modifies T Cell Populations in Nasal Biopsies}

Pre- and Day 4 post-LAIV nasal biopsies were collected from nonsmokers and smokers and processed for flow cytometry. CD3+CD45+CD4-CD8- T cells as percent of total nasal biopsy cells increased in nonsmokers $(\mathrm{P}=0.008$, Wilcoxon signed rank test $)$ but not smokers $(\mathrm{P}=0.300)$ (Figure $1 \mathrm{G})$. These CD3 T cells were neither helper (CD4) nor cytotoxic (CD8) T cells. To identify these non-classical T cells, pan $\gamma \delta$ T cell receptor (TCR) expression was evaluated. Only the subset of individuals with both pre and post LAIV $\gamma \delta$ TCR expression data ( $\mathrm{N}=5$, nonsmokers and $\mathrm{N}=8$ smokers) were included in the analysis. A representative flow cytometric histogram details the $\gamma \delta$ TCR expression relative to isotype control (Figure 2A). Similar to the pattern for CD3+ T cells, delta change in $\gamma \delta \mathrm{T}$ cell percentages (Figure 2B) was suppressed in smokers compared to nonsmokers $(\mathrm{P}=$ 0.045) within the sample subset..

\section{Discussion}

In superficial nasal mucosal biopsies in human volunteers, we have identified a cell population with characteristics of $\gamma \delta \mathrm{T}$ cells which appears to increase several days 
following LAIV inoculation. This is a novel finding and is the first evidence that $\gamma \delta \mathrm{T}$ cells may participate in early influenza-induced mucosal responses in humans. $\gamma \delta$ T lymphocytes or intraepithelial lymphocytes are at their highest concentration in epithelial tissues and have been identified in bronchial biopsies in individuals at baseline [11]. Their location in mucosal epithelia allows them to quickly respond to virus infections, and $\gamma \delta \mathrm{T}$ cells sense "danger" from nearby epithelial cells through recognition of stressed cell ligands that are upregulated by viral infections [12] and from activation by virus-induced proinflammatory cytokines in the epithelial microenvironment [13]. $\gamma \delta \mathrm{T}$ cells migrate to the lung following influenza infection in response to chemokines $[14,15]$, which supports the LAIV-induced responses observed here. During an influenza infection, $\gamma \delta$ T cells could kill virus infected epithelial cells similar to cytotoxic T and NK cells [13] and also could produce cytokines such as IFN $\gamma$, IL-17, and MCP-1 to induce immune cell chemotaxis [13]. We have previously shown both that cytotoxic NK cell activity [16] as well as IFN $\gamma$ levels [3] are suppressed in smokers following LAIV inoculation. Therefore, because $\gamma \delta$ T cells and NK cells share functional redundancy, deficiencies in $\gamma \delta \mathrm{T}$ cell as well as NK cell activity could contribute to the suppression of LAIV-induced IFN $\gamma$ secretion observed in smokers. One limitation of our study was the small sample subset available for analysis of $\gamma \delta$ TCR expression. Initial studies were designed to follow classical CD4 and CD8 T cells before and after LAIV inoculation. However, our results revealed a non-classical CD3 T cell population that necessitated altering our protocol to include analysis of $\gamma \delta$ T cells. Limitations arising from the small subset used to assess $\gamma \delta \mathrm{T}$ cell populations within this study could be addressed by a larger more definitive study that fully explores $\gamma \delta \mathrm{T}$ cell activation markers, specific TCR subsets, and $\gamma \delta$ T cell-secreted cytokines.

We did not observe significant populations of CD4 or CD8 T cells in the nasal mucosa at baseline. This is in contrast to another report that identifies CD8+ $\alpha / \beta \mathrm{T}$ cells as the main phenotype of intraepithelial nasal mucosal T cells [17]. However, subjects in that cohort underwent nasal surgery to obtain tissue samples whereas the subjects here underwent superficial nasal scrapes. It is likely the depth of the biopsy impacts the phenotype of intraepithelial lymphocytes recovered. We also did not observe increases in conventional $\mathrm{T}$ cell percentages by day 4 post LAIV. This is likely because influenza specific lymphocytes such as CD8 T cells do not migrate to the airways until day 7 post infection [18]. Our protocol was designed to characterize early host defense processes after virus inoculation, but modifying our LAIV protocol to include later time points may capture influx of influenza specific CD4 or CD8 T cells.

There are conflicting data exploring the effects of smoking on respiratory mucosal $\mathrm{T}$ cell function in humans. Several groups have indicated that both smokers and smokers with COPD had increased CD3+CD8+ T cell percentages in bronchial biopsies [19] as well as lung parenchyma [20] at baseline compared to never smokers. However, other researchers have demonstrated that sputum CD8+ T lymphocytes are decreased in smokers before smoking cessation compared to after cessation and never smokers [21]. In addition, these CD8+ T cells were different compared to the $\gamma \delta \mathrm{T}$ cell population described in this study, although increased $\gamma \delta \mathrm{T}$ cell percentages have been identified in the BAL of smokers [22]. In addition, smokers with normal lung function had increased expression of CD4+CD25+ regulatory T cells within the BAL compared to never-smokers [23]. Therefore, while it is likely that smokers exhibit constitutive inflammation in the lower airways characterized by cytotoxic $\mathrm{T}$ cell infiltration, it is unclear what effect smoking may have on cytotoxic $\mathrm{T}$ cells in the nasal mucosa. 


\section{Conclusions}

Cigarette smoking may inhibit virus-induced responses of $\gamma \delta \mathrm{T}$ cells following inoculation with LAIV. Understanding the role of cigarette smoke-induced suppression of $\gamma \delta \mathrm{T}$ cells in the context of immune responses within the nasal mucosa could provide novel therapeutic targets. In addition, the in vivo $\mathrm{T}$ cell assays used to identify and characterize a relatively rare intra-mucosal immune cell type from human biopsy samples, which can be obtained through non-invasive procedures as shown here, could prove a useful technique to examine immunotoxicological responses of other inhaled agents.

\section{Highlights}

We have identified $\gamma \delta$ T cells infiltrating into the nasal mucosa in response to LAIV. We show that infiltration of $\gamma \delta$ T cells is suppressed in smokers.

Flow cytometry of can be used to characterize nasal mucosal immune cell phenotypes.

\section{References}

1. Horvath KM, Brighton LE, Zhang W, Carson JL, Jaspers I. Epithelial Cells From Smokers Modify Dendritic Cell Responses in the Context of Influenza Infection. Am J Respir Cell Mol Biol. 2010

2. Jaspers I, Horvath KM, Zhang W, Brighton LE, Carson JL, Noah TL. Reduced Expression of IRF7 in Nasal Epithelial Cells from Smokers after Infection with Influenza. Am J Respir Cell Mol Biol. 2009

3. Noah TL, Zhou H, Monaco J, Horvath K, Herbst M, Jaspers I. Tobacco Smoke Exposure and Altered Nasal Responses to Live Attenuated Influenza Virus. Environ Health Perspect. 2010

4. MacKenzie JS, MacKenzie IH, Holt PG. The effect of cigarette smoking on susceptibility to epidemic influenza and on serological responses to live attenuated and killed subunit influenza vaccines. J Hyg (Lond). 1976; 77:409-417. [PubMed: 1069819]

5. Pyhala R, Takala J, Turunen A, Aho K. Smoking and influenza in the elderly: a seroepidemiological study. Eur J Respir Dis. 1983; 64:212-216. [PubMed: 6840234]

6. Mancini DA, Mendonca RM, Mendonca RZ, do Prado JA, Andrade Cde M. Immune response to vaccine against influenza in smokers, non-smokers and, in individuals holding respiratory complications. Boll Chim Farm. 1998; 137:21-25. [PubMed: 9595830]

7. Boyce TG, Gruber WC, Coleman-Dockery SD, Sannella EC, Reed GW, Wolff M, Wright PF. Mucosal immune response to trivalent live attenuated intranasal influenza vaccine in children. Vaccine. 1999; 18:82-88. [PubMed: 10501238]

8. Maassab HF, Bryant ML. The development of live attenuated cold-adapted influenza virus vaccine for humans. Rev Med Virol. 1999; 9:237-244. [PubMed: 10578119]

9. Hammitt LL, Bartlett JP, Li S, Rahkola J, Lang N, Janoff EN, Levin MJ, Weinberg A. Kinetics of viral shedding and immune responses in adults following administration of cold-adapted influenza vaccine. Vaccine. 2009; 27:7359-7366. [PubMed: 19800447]

10. Lau YF, Santos C, Torres-Velez FJ, Subbarao K. The magnitude of local immunity in the lungs of mice induced by live attenuated influenza vaccines is determined by local viral replication and induction of cytokines. J Virol. 2011; 85:76-85. [PubMed: 20962087]

11. Wisnewski AV, Cain H, Magoski N, Wang H, Holm CT, Redlich CA. Human gamma/delta T-cell lines derived from airway biopsies. Am J Respir Cell Mol Biol. 2001; 24:332-338. [PubMed: 11245633]

12. Steinle A, Li P, Morris DL, Groh V, Lanier LL, Strong RK, Spies T. Interactions of human NKG2D with its ligands MICA, MICB, and homologs of the mouse RAE-1 protein family. Immunogenetics. 2001; 53:279-287. [PubMed: 11491531]

13. Bonneville M, O'Brien RL, Born WK. Gammadelta T cell effector functions: a blend of innate programming and acquired plasticity. Nat Rev Immunol. 2010; 10:467-478. [PubMed: 20539306] 
14. Carding SR. A role for gamma/delta T cells in the primary immune response to influenza virus. Res Immunol. 1990; 141:603-606. [PubMed: 2151346]

15. Eichelberger M, Allan W, Carding SR, Bottomly K, Doherty PC. Activation status of the CD4-8gamma delta-T cells recovered from mice with influenza pneumonia. J Immunol. 1991; 147:20692074. [PubMed: 1833449]

16. Horvath KM, Herbst M, Zhou H, Zhang H, Noah TL, Jaspers I. Nasal lavage natural killer cell function is suppressed in smokers after live attenuated influenza virus. Respir Res. 2011; 12:102. [PubMed: 21816072]

17. Jahnsen FL, Farstad IN, Aanesen JP, Brandtzaeg P. Phenotypic distribution of T cells in human nasal mucosa differs from that in the gut. Am J Respir Cell Mol Biol. 1998; 18:392-401. [PubMed: 9490657]

18. Verbist KC, Cole CJ, Field MB, Klonowski KD. A role for IL-15 in the migration of effector CD8 $\mathrm{T}$ cells to the lung airways following influenza infection. J Immunol. 2011; 186:174-182. [PubMed: 21098221]

19. Roos-Engstrand E, Pourazar J, Behndig AF, Blomberg A, Bucht A. Cytotoxic T cells expressing the co-stimulatory receptor NKG2 D are increased in cigarette smoking and COPD. Respir Res. 2010; 11:128. [PubMed: 20863413]

20. Olloquequi J, Ferrer J, Montes JF, Rodriguez E, Montero MA, Garcia-Valero J. Differential lymphocyte infiltration in small airways and lung parenchyma in COPD patients. Respir Med. 2010; 104:1310-1318. [PubMed: 20359875]

21. Bouloukaki I, Tsoumakidou M, Vardavas CI, Mitrouska I, Koutala E, Siafakas NM, Schiza SE, Tzanakis N. Maintained smoking cessation for 6 months equilibrates the percentage of sputum CD8+ lymphocyte cells with that of nonsmokers. Mediators Inflamm. 2009; 2009812102.

22. Roos-Engstrand E, Ekstrand-Hammarstrom B, Pourazar J, Behndig AF, Bucht A, Blomberg A. Influence of smoking cessation on airway T lymphocyte subsets in COPD. COPD. 2009; 6:112120. [PubMed: 19378224]

23. Roos-Engstrand E, Pourazar J, Behndig AF, Bucht A, Blomberg A. Expansion of CD4+CD25+ helper T cells without regulatory function in smoking and COPD. Respir Res. 2011; 12:74. [PubMed: 21651772] 
A

D
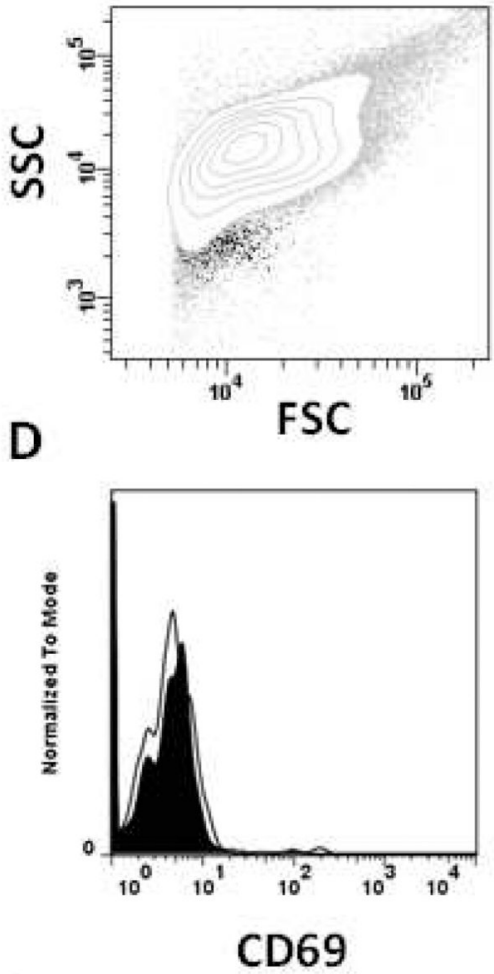

B

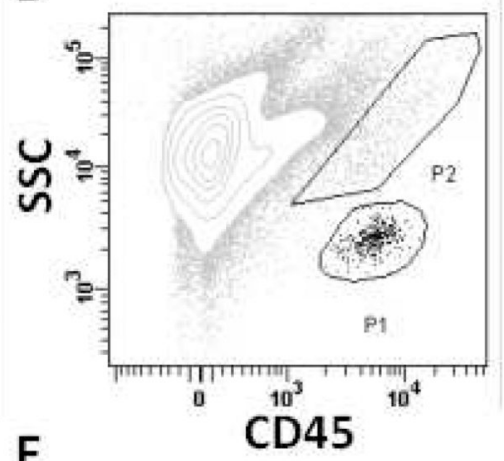

E

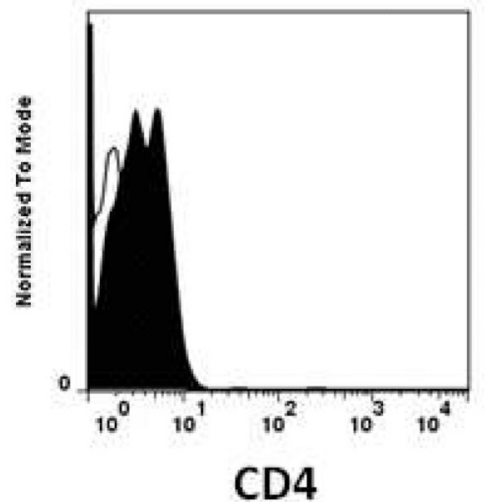

C

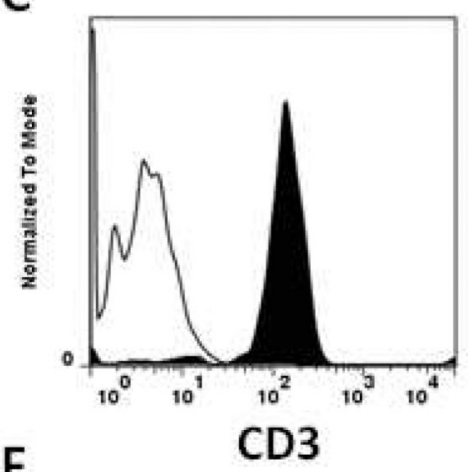

F

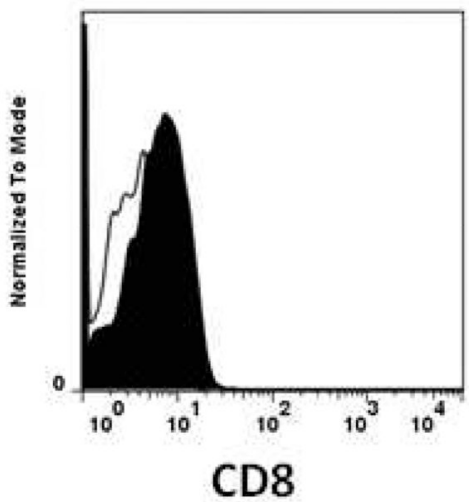

G

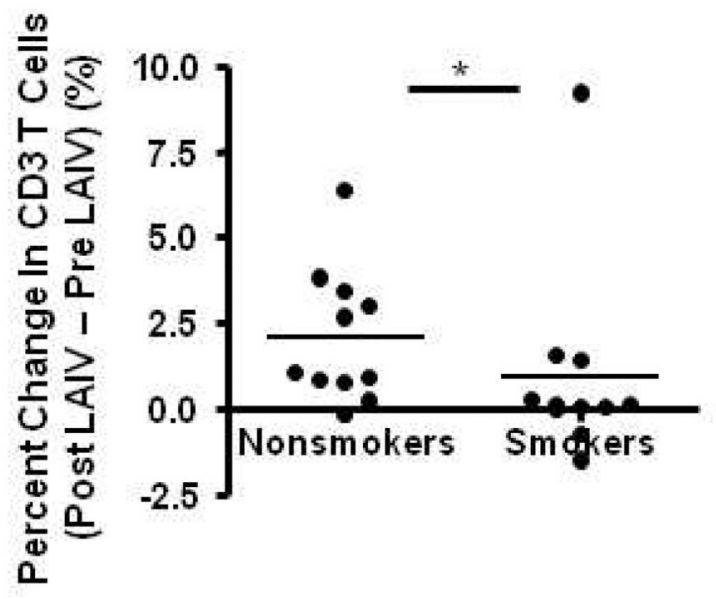

Figure 1.

CD45+CD3+ Lymphocyte Populations in Nonsmokers and Smokers At Baseline and Following LAIV. Nasal biopsies obtained on screen day were processed and stained with immune markers for flow cytometric analysis. A) Forward scatter (FSC) and side scatter (SSC) fluorescence. B) Nasal leukocytes were identified based on SSC voltages and positive staining for CD45 in gate P1. Expression of immune markers on CD45+ cells for C) CD3, D) $\mathrm{CD} 69$, E) CD4, and F) CD8. G) CD3+CD4-CD8- T lymphocyte responses are determined by subtracting pre LAIV (baseline) percentages from post LAIV T cell percentages. $* \mathrm{P}<0.05$ (Mann Whitney test) Open histograms = isotype controls, black 
histograms $=$ surface marker staining. A representative image of flow cytometric staining in normal individuals at baseline is shown. 
A B
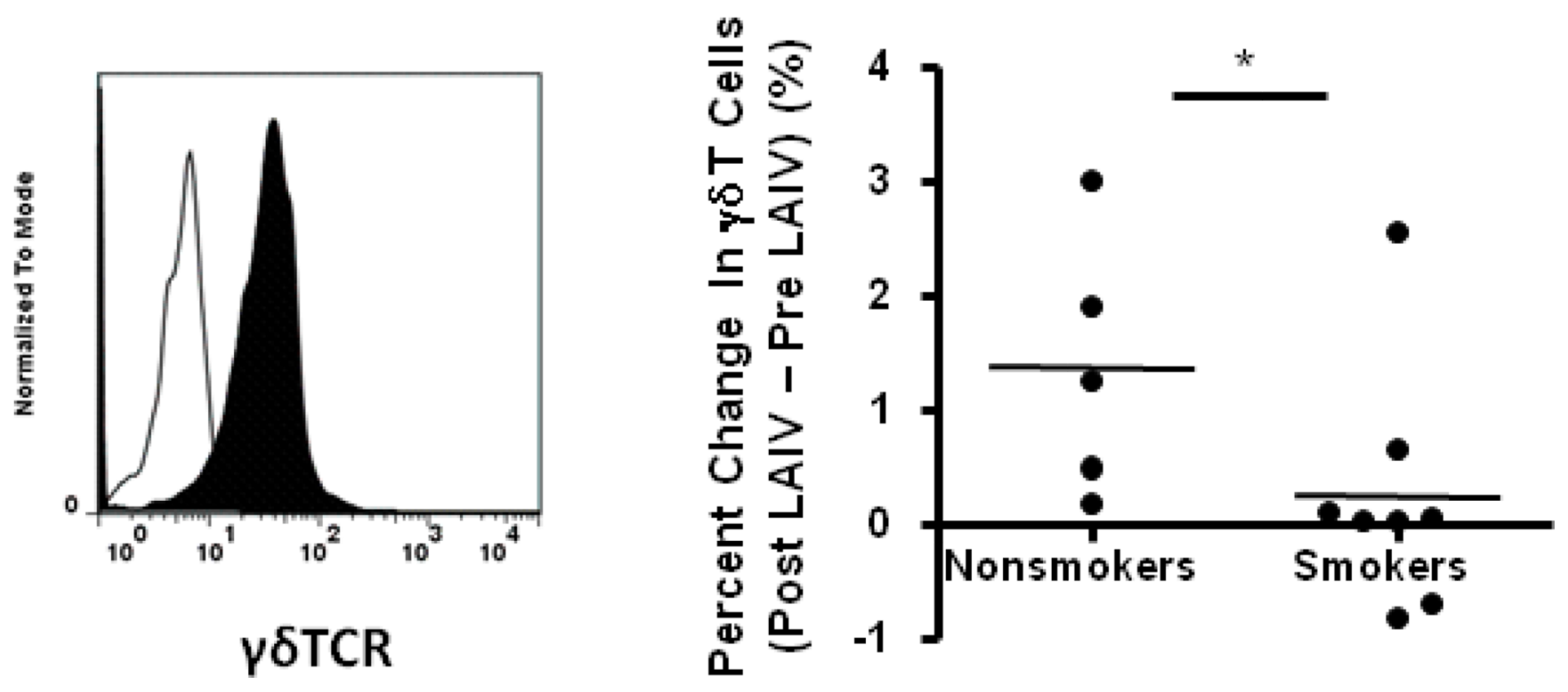

Figure 2.

$\gamma \delta$ TCR Expression on Nasal Lymphocytes. CD45+CD3+ lymphocytes were evaluated for $\gamma \delta$ TCR expression in a subset of individuals. A) A representative image shows istoype control staining (open histogram) and $\gamma \delta$ TCR staining (black histogram) on CD45+CD3+CD4-CD8- lymphocytes. B) $\gamma \delta$ T lymphocyte responses are determined by subtracting pre LAIV (baseline) percentages from post LAIV percentages for CD3+CD4-CD8 - T cells. * $\mathrm{P}<0.05$ (Mann Whitney test). 